\title{
Plant responses and tolerance to high temperature stress: role of exogenous phytoprotectants
}

\begin{abstract}
Among the abiotic stresses high temperature stress is one of the most detrimental stresses threatening higher plant productivity and survival throughout the world. Each degree Celsius increase of average growing season temperature may decrease crop yield and affect plant distribution. On the other hand, global average temperatures are supposed to increase from 1.8 to $4.0^{\circ} \mathrm{C}$ or higher by 2100 as compared to the $1980 \mathrm{I} 2000$ average. Plants are intimidated by adverse effects of high temperature stresses. Protein denaturation, inactivation of enzymes, production of reactive oxygen species, and disruption of membrane structure are some of the primary damage effects of high temperature that are also responsible for damage of ultrastructural cellular components. These anomalies hamper plant growth and development. Although higher plants develop their own defense strategies to overcome the high temperature stress effects, these often are not enough, therefore substantial damage is observed. The metabolism in plants is altered in response to high temperature stress. The antioxidants, secondary metabolites, hormones, osmoprotectants, and many other essential biomolecules are modulated, which help to defend against high temperature impacts. Moreover, numerous studies have proved that as protectants the exogenously applied hormones, osmoregulators, antioxidants, signaling molecules, polyamines, and trace elements confer high temperature stress tolerance in the organisms. This chapter presents the responses of plants to high temperature stress and evaluates the role of exogenous protectants under high temperature stress.
\end{abstract}

Keyword: Phytoprotectants; Thermotolerance; Abiotic stress; Climate change 